\title{
SATELLITES OF HALF EXACT FUNCTORS, A CORRECTION
}

\section{HARLEY FLANDERS}

In Chapter III of H. Cartan and S. Eilenberg, Homological algebra, there is the substantial

THEOREM 3.1. Let

$$
0 \rightarrow A^{\prime} \rightarrow A \rightarrow A^{\prime \prime} \rightarrow 0
$$

be an exact sequence. If $T$ is a covariant half exact functor then the sequence

$$
\begin{aligned}
\cdots & \rightarrow S^{n-1} T\left(A^{\prime \prime}\right) \rightarrow S^{n} T\left(A^{\prime}\right) \rightarrow S^{n} T(A) \\
& \rightarrow S^{n} T\left(A^{\prime \prime}\right) \rightarrow S^{n+1} T\left(A^{\prime}\right) \rightarrow \cdots
\end{aligned}
$$

is exact. For $T$ contravariant, $A^{\prime}$ and $A^{\prime \prime}$ should be interchanged.

The proof, which occupies pp. 40-42 of the book, is routine until the critical step of showing that the sequence $S_{1} T\left(A^{\prime \prime}\right) \rightarrow T\left(A^{\prime}\right) \rightarrow T(A)$ is exact. The proof of this assertion begins with the last paragraph of p. 41 and goes through most of p. 42 .

At Cambridge University in 1957 (on a National Science Foundation fellowship) we observed that this part of the proof contains an error. Fortunately it can be made right, as we communicated to Professor Eilenberg at the time. Since we have been asked for this correction several times it seems proper to put it in print.

The precise mistake is the sentence on lines $12-15$ of p. 42 . In the special case being considered, the diagram on p. 41 reduces to

$$
\begin{aligned}
& 00 \\
& \downarrow \downarrow \downarrow \\
& 0 \rightarrow M \rightarrow M \longrightarrow 0 \\
& \downarrow \downarrow_{\lambda} \downarrow \phi^{\prime} \\
& 0 \rightarrow M \stackrel{\lambda}{\rightarrow} R \rightarrow P \longrightarrow 0 \\
& \downarrow \quad \downarrow \quad \downarrow \phi
\end{aligned}
$$

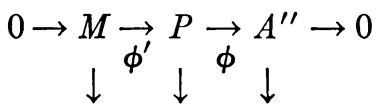

$$
\begin{aligned}
& 0 \quad 0 \quad 0 \text {. }
\end{aligned}
$$

Received by the editors April 26, 1963. 
Here $R$ is the submodule of $P+P$ consisting of all $\left(p_{1}, p\right)$ with $\phi p_{1}=\phi p$, i.e., $p_{1} \equiv p\left(\bmod \phi^{\prime} M\right)$. Also $\lambda(m)=\left(\phi^{\prime} m, 0\right), \mu(m)=\left(0, \phi^{\prime} m\right)$. From the first two rows one has the diagram on top of p. 42 , for this special case:

$$
\begin{array}{ccc}
0 \longrightarrow & T(M) \stackrel{\iota}{\longrightarrow} T(M) \longrightarrow 0 \\
\downarrow & \downarrow T(\mu) & \downarrow \\
0 \longrightarrow T(M) \underset{T(\lambda)}{\longrightarrow} T(R) \longrightarrow T(P) .
\end{array}
$$

According to the construction of connecting homomorphisms, this yields

$$
\bar{u}: \operatorname{Ker}(T(M) \rightarrow T(P))=S_{1} T\left(A^{\prime \prime}\right) \rightarrow T(M)
$$

with the commutative diagram

$$
\begin{aligned}
& 0 \longrightarrow S_{1} T\left(A^{\prime \prime}\right) \longrightarrow T(M) \\
& \downarrow \bar{u} \downarrow T(\mu) \\
& T(M) \underset{T(\lambda)}{\longrightarrow} T(R) .
\end{aligned}
$$

Considering the distinction between $\lambda$ and $\mu$ one cannot conclude that $\bar{u}$ coincides with the inclusion map in the first row.

Here is the correction. We begin with the commutative diagram with exact rows

$$
\begin{aligned}
& 0 \longrightarrow M \stackrel{\psi^{\prime}}{\longrightarrow} P \stackrel{\psi}{\longrightarrow} A^{\prime \prime} \longrightarrow 0 \\
& \downarrow f^{\prime} \quad \downarrow f \quad \downarrow \downarrow \\
& 0 \longrightarrow A^{\prime} \underset{\phi^{\prime}}{\longrightarrow} A \underset{\phi}{\longrightarrow} A^{\prime \prime} \longrightarrow 0
\end{aligned}
$$

which leads to

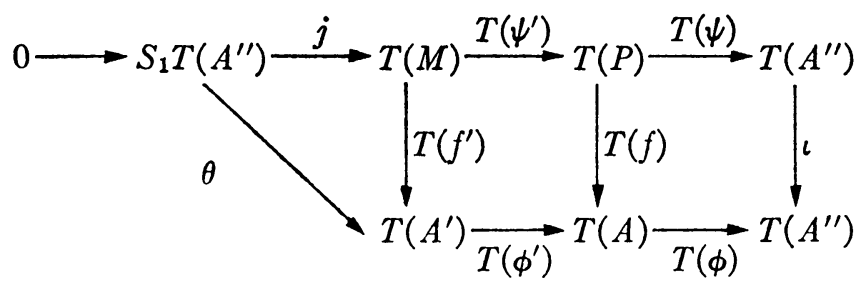

As is done on p. 41, we construct the commutative diagram with exact rows and columns: 


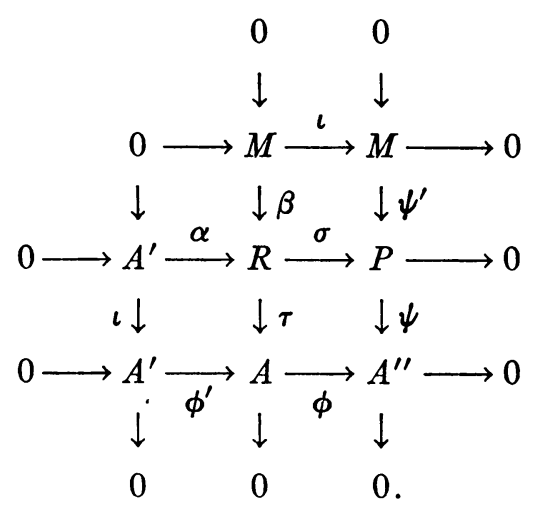

The module $R$ is the kernel of $(a, p) \rightarrow \phi a-\psi p$ so that $R \leqq A+P$ and the various new mappings are given by

$$
\begin{aligned}
\alpha a^{\prime} & =\left(\phi^{\prime} a^{\prime}, 0\right), \quad \beta m=\left(0, \psi^{\prime} m\right), \\
\sigma(a, p) & =p, \quad \tau(a, p)=a .
\end{aligned}
$$

Because $P$ is projective, the middle row splits.

Now what we do is to define a mapping

$$
\delta: R \rightarrow A^{\prime} \text {. }
$$

If $(a, p) \in R, \phi a=\psi p$, then $\phi(a-f p)=0$ and so $a-f p=\phi^{\prime} a^{\prime}$ for a unique $a^{\prime} \in A^{\prime}$. We set

$$
\delta(a, p)=a^{\prime} .
$$

We note the properties of this $\delta$ :

$$
\begin{gathered}
\delta \alpha=\iota, \\
\delta \beta=-f^{\prime} .
\end{gathered}
$$

For $\delta \alpha\left(a^{\prime}\right)=\delta\left(\phi^{\prime} a^{\prime}, 0\right)=a^{\prime} ;$ and $\delta \beta(m)=\delta\left(0, \psi^{\prime} m\right)=a^{\prime}$ where $\phi^{\prime} a^{\prime}$ $=0-f \psi^{\prime} m=-\phi^{\prime} f^{\prime} m$, i.e., $a^{\prime}=-f^{\prime} m, \delta \beta=-f^{\prime}$.

We are now ready to establish

$$
\operatorname{Ker} T\left(\phi^{\prime}\right) \leqq \operatorname{Im}(\theta) .
$$

Let $x \in T\left(A^{\prime}\right)$ and $T\left(\phi^{\prime}\right) x=0$. Then $T(\tau) T(\alpha) x=0$ so that there exists $y \in T(M)$ such that $T(\alpha) x=T(\beta) y$. (Here we use the exactness of $T(M) \rightarrow T(R) \rightarrow T(A)$ which is a consequence of the half exactness of $T$.) Consequently

$$
\begin{aligned}
T(\delta) T(\alpha) x & =T(\delta) T(\beta) y, \\
x & =-T\left(f^{\prime}\right) y .
\end{aligned}
$$


We also have

so that

$$
T\left(\psi^{\prime}\right) y=T(\sigma) T(\beta) y=T(\sigma) T(\alpha) x=0
$$

$$
\begin{aligned}
& y \in \operatorname{Ker} T\left(\psi^{\prime}\right)=\operatorname{Im}(j), \\
& y=j z, \quad z \in S_{1} T\left(A^{\prime \prime}\right), \\
& x=-T\left(f^{\prime}\right) j z=\theta(-z) .
\end{aligned}
$$

For the dual situation, our large diagram is replaced by

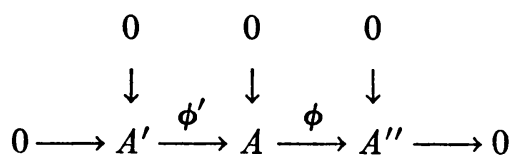

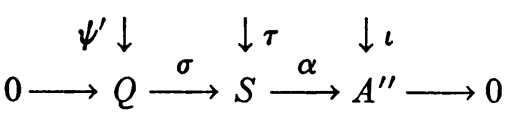

$$
\begin{aligned}
& \begin{array}{llll}
\downarrow \downarrow & \downarrow \beta & \downarrow \\
& \stackrel{\iota}{\longrightarrow} N \longrightarrow 0
\end{array} \\
& \downarrow \quad \downarrow \\
& 0 \quad 0 \text {. }
\end{aligned}
$$

This time $S=\operatorname{Coker}\left(A^{\prime} \rightarrow A+Q\right)=$ Coker $\lambda$ where the mapping is $\lambda: a^{\prime} \rightarrow\left(\phi^{\prime} a^{\prime},-\psi^{\prime} a^{\prime}\right)$. The other mappings are

$$
\begin{aligned}
& \sigma q=(0, q)+\operatorname{Im} \lambda, \\
& \tau a=(a, 0)+\operatorname{Im} \lambda, \\
& \alpha[(a, q)+\operatorname{Im} \lambda]=\phi(a), \\
& \beta[(a, q)+\operatorname{Im} \lambda]=\psi(q) .
\end{aligned}
$$

The trick is to define $\delta: A^{\prime \prime} \rightarrow S$ by

$$
\delta a^{\prime \prime}=(a,-f a)+\operatorname{Im} \lambda \quad \text { where } \phi a=a^{\prime \prime} .
$$

Here, of course, $Q$ is injective and we have

$$
\begin{aligned}
0 \longrightarrow & A^{\prime} \stackrel{\phi^{\prime}}{\longrightarrow} A \stackrel{\phi}{\longrightarrow} A^{\prime \prime} \rightarrow 0 \\
& \downarrow \downarrow \quad f \downarrow \quad f^{\prime \prime} \downarrow \\
0 \longrightarrow & A^{\prime} \underset{\psi^{\prime}}{\longrightarrow} Q \underset{\psi}{\longrightarrow} N \longrightarrow 0 .
\end{aligned}
$$

The rest offers no difficulty. 